\title{
Übersichten
}

Hautarzt $2021 \cdot 72: 321-327$

https://doi.org/10.1007/s00105-020-04720-1

Online publiziert: 11 . November 2020

(c) Der/die Autor(en) 2020

\author{
Johannes Wohlrab ${ }^{1,2}$ • Ulrich Mrowietz ${ }^{3}$ Stephan Weidinger ${ }^{3}$ Thomas Werfel ${ }^{4}$. \\ Andreas Wollenberg ${ }^{5}$ \\ 'Universitätsklinik und Poliklinik für Dermatologie und Venerologie, Martin-Luther-Universität Halle- \\ Wittenberg, Halle (Saale), Deutschland \\ ${ }^{2}$ An-Institut für angewandte Dermatopharmazie, Martin-Luther-Universität Halle-Wittenberg, Halle- \\ Wittenberg, Deutschland \\ ${ }^{3}$ Klinik für Dermatologie, Venerologie und Allergologie, Christian-Albrechts-Universität Kiel, Kiel, \\ Deutschland \\ ${ }^{4}$ Klinik für Dermatologie und Allergologie, Medizinische Hochschule Hannover, Hannover, Deutschland \\ ${ }^{5}$ Klinik für Dermatologie und Allergologie, Ludwig-Maximilian-Universität München, München, \\ Deutschland
}

\section{Handlungsempfehlung zur Therapieumstellung von Immunsuppressiva auf Dupilumab bei Patienten mit atopischer Dermatitis}

(AZA) [14-17] oder Mycophenolsäurederivate (MPA) [18] eingesetzt werden. Für ein chronisches Handekzem im Rahmen des atopischen Syndroms kann auch eine Therapie mit Alitretinoin (ALI) erwogen werden [19].

Es gibt verschiedene Szenarien, die dazu führen können, dass eine immunsuppressive Systemtherapie mit den genannten kleinmolekularen Arzneistoffen beendet und auf eine zielgerichtete Therapie mit dem therapeutischen Antikörper Dupilumab umgestellt werden muss. Für das dabei aus pharmakologischer und regulatorischer Sicht notwendige Management gibt es aber aktuell nur sehr pauschale und wenig differenzierte Handlungsempfehlungen bzw. systematische Entscheidungshilfen [20]. Diese wurden nun durch eine Expertengruppe bearbeitet und sollen im Folgenden verfügbar gemacht werden.

\section{Pharmakologische Kenndaten relevanter Arzneistoffe}

Arzneistoffe mit Zulassung in der Indikation „atopische Dermatitis"

Für die Systemtherapie der atopischen Dermatitis sind in Deutschland nur SGK, CSA und Dupilumab zugelassen [21]. Beim Einsatz von SGK und CSA sollte wegen der immunsuppressiven Wirkung ein erhöhtes Risiko für Infektionen beachtet werden [22]. Ergänzend dazu gilt eine Kontraindikation für Lebendimpfstoffe. Zudem sollte der Einsatz bei manifesten oder anamnestischen Malignomen vermieden werden. Es liegt für keinen der genannten Wirkstoffe eine Zulassung für den Einsatz in der Schwangerschaft oder Stillzeit vor. Aufgrund umfangreicher Daten kann aber der Einsatz, insbesondere von CSA, nach einer individuellen Risikoabschätzung und mit Einverständnis der Patientin erwogen werden [23].

\section{Glukokortikoide}

BeiSGKhandelt es sich um lipophile Hormone, die eine genomische (zytosolische 
Rezeptorbindung nach transmembranöser Diffusion) und eine nichtgenomische Wirkung (Interaktionen mit Membranen) entfalten. Die empfohlene Tagesdosis von SGK bei atopischer Dermatitis ist nicht einheitlich und richtet sich nach der Schwere der Erkrankung und möglicher Komorbidität bzw. Komedikation. Im klinischen Alltag hat sich eine Dosierung von $0,5-1,0 \mathrm{mg} / \mathrm{kgKG}$ [Körpergewicht]/Tag Prednisolonäquivalent bis maximal 14 Tage bewährt, um ein Ausschleichmanöver zur Dosisreduktion zu vermeiden. Eine Anwendung für den mittel- und langfristigen Gebrauch bei atopischer Dermatitis kann wegen der unerwünschten Arzneimittelwirkungen (UAWs) nicht empfohlen werden [5]. So geht der Einsatz von SGK selbst bei niedrig dosierter Anwendung regelmäßig mit unerwünschten Effekten einher. Das Ausmaß und die Reversibilität sind interindividuell sehr verschieden und stark abhängig von Dosierung, Anwendungsdauer und Applikationsform. Unter Langzeitanwendung zeigen sich Suppressionsphänomene der adrenalen Achse, Störungen des Kalziumstoffwechsels mit Osteoporose, zentralnervöse Symptome, Myopathie, Blutbildveränderungen, Wachstumsretardierung im Kindesalter, Kataraktentwicklung, Steroiddiabetes sowie gastrointestinale Ulkusbildung $[24,25]$. Sollte eine mittel- und langfristige Systemtherapie notwendig sein, so empfiehlt sich leitliniengerecht entweder die Einleitung einer CSA- oder einer Dupilumab-Therapie [26, 27].

\section{Ciclosporin}

Ciclosporin (CSA) ist ein stark lipophiles zyklisches Polypeptid. Es bindet nach Diffusion durch die Zellmembran (z.B. Lymphozyten) an das zytoplasmatische Rezeptormolekül Cyclophilin (Familie der Immunophiline), inhibiert dadurch die kalziumabhängige Phosphatase Calcineurin und damit die Aktivierung eines nukleären Faktors aktivierter T-Zellen (NFAT), der nach Translokation in den Zellkern die Genaktivierung und Transkription von proinflammatorischen Zytokinen (z.B. IL[Interleukin]-2, TNF[TumorNekrose-Faktor]- $\alpha$, IFN[Interferon]- $\gamma$ ) reguliert [28]. Es wird typischerwei- se in einem Dosisbereich von 2,5-5 $(7,5) \mathrm{mg} / \mathrm{kgKG} / \mathrm{Tag}$ in 2 Einzeldosen per os verabreicht. Durch den früheren intensiven Einsatz von CSA in der Transplantationsmedizin liegen umfangreiche pharmakologische Daten und Erfahrungen vor. Dies betrifft auch den Einsatz in der Langzeitanwendung, im Kindesalter und in der Schwangerschaft. Wegen der Metabolisierung von CSA in Leber und Darm durch CYP3A4 sowie der Abhängigkeit der Clearance von P-Glykoprotein treten Arzneimittelinteraktionen mit einer Vielzahl anderer Wirksubstanzen (z.B. Azolantimykotika, Antiepileptika, Terbinafin) auf [29].

Neben einer stark eingeschränkten Nierenfunktion, dem Vorliegen von Malignomen (auch anamnestisch) und einem ausgeprägten arteriellen Hypertonus gelten v. a. schwere Infektionserkrankungen als absolute Kontraindikation. Die kombinierte Exposition mit UVALicht (natürliche oder künstliche Bestrahlung) oder die einer Ciclosporin vorausgehenden PUVA-Therapie gelten als Risikofaktor für die Entstehung von nichtmelanozytärem Hautkrebs [30-32].

Unter der Therapie werden gelegentlich nach einer Therapiedauer von $>2$ Jahren häufig Nierenfunktionsstörungen und die Ausbildung eines arteriellen Hypertonus beobachtet [31, 33-35]. Seltener treten eine Gingivahyperplasie oder eine Hypertrichose auf [36]. Die Verträglichkeit im Kindesalter scheint besser zu sein als bei Erwachsenen [37-39]. Das in der Transplantationsmedizin indizierte „drug monitoring“ ist in der Dermatologie pharmakologisch weder notwendig noch sinnvoll und wird nur zur Überprüfung der Therapieadhärenz genutzt [40].

\section{Dupilumab}

Mit Dupilumab steht für erwachsene Patienten seit 2017 und für Jugendliche (ab 12. Lebensjahr) seit 2019 ein therapeutischer Antikörper für die zielgerichtete Therapie der mittelschweren bis schweren $\mathrm{AD}$ zur Verfügung. Er bindet an die $\alpha$-Untereinheit des IL-4-Rezeptors und inhibiert damit die Aktivierung der intrazellulären Signalkaskade von IL-4 und IL-13 [41]. Dupilumab ist ein $\operatorname{Ig}($ Immunglobulin)G-Mole- kül, das ca. 7 bis 10 Tage nach subkutaner Applikation die höchste Serumkonzentration erreicht und eine absolute Bioverfügbarkeit von ca. $64 \%$ aufweist. Es unterliegt wie alle IgG-Moleküle einer Clearance, die durch lineare und nichtlineare Eliminationsprozesse bedingt wird. Neben der zielantigenvermittelten Elimination (sog. „antigen sink") und einer geringen Proteolyserate in der Leber erfolgt die Elimination v. a. über das retikuloendotheliale System in der Interaktion mit Makrophagen und Monozyten sowie durch unspezifische Pino-/Endozytose von Endothelzellen, die auch in Abhängigkeit von der Expression des $\mathrm{nFcR}$ (Brambell-Rezeptor) einen Recycling-Prozess steuern [42]. Eine Bildung von endogenen Antikörpern gegen Dupilumab (sog. ,anti drug antibodies" [ADA]) mit neutralisierender Wirkung findet sich nur in einem sehr geringen und klinisch nicht relevanten Ausmaß von ca. 0,5-2\% [43]. Da bei der Anwendung von Dupilumab keine direkten Arzneimittelinteraktionen mit einem der bereits genannten „small molecules“ zu erwarten sind, bestehen aus pharmakologischer Sicht keine Bedenken gegen eine unmittelbare, indikationsgerechte Anschlusstherapie bzw. eine kombinierte Anwendung von Dupilumab mit einem konventionellen Immunsuppressivum wie SGK, CSA, MTX, AZA oder MPA. Die vorliegenden klinischen Studiendaten mit Dupilumab (SOLO1 und 2, CHRONOS) weisen bei der Mehrzahl der Patienten einen soliden therapeutischen Effekt (klinische relevante Reduktion von EASI [Eczema Area and Severity Index]/SCORAD [Scoring Atopic Dermatitis] bereits nach ca. 4 bis 6 Wochen) aus. Eine klinisch relevante Reduktion des Juckreizes wird häufig schon früher beobachtet [44-46]. Auch wenn die Ansprechraten im weiteren Therapieverlauf noch leicht ansteigen, so kann bei der Mehrzahl der Patienten davon ausgegangen werden, das nach dieser Initialphase der Dupilumab-Anwendung ein relevantes klinisches Ansprechen eintritt [43, 47]. Dupilumab wird häufig bei $\mathrm{Pa}$ tienten initiiert, die sich noch unter einer Therapie mit Immunsuppressiva, insbesondere CSA, befinden. Für eine solche Therapieumstellung von CSA aufDupilu- 
mab kann eine kombinierte Anwendung für 4 bis 6 Wochen erwogen werden. Für eine längerfristige kombinierte Anwendung eines konventionellen Immunsuppressivums mit Dupilumab gibt es bisher keine Evidenz.

\section{Arzneistoffe, die in der Indikation atopische Dermatitis "off label" angewendet werden}

\section{Methotrexat}

Das Zytostatikum MTX wird nach aktiver Aufnahme in die Zelle glutamyliert und hemmt die Dihydrofolatreduktase (Antifolat), die als Schlüsselenzym der Folsäuresynthese fungiert und somit eine Voraussetzung für die RNA(Ribonukleinsäure)- und DNA(Desoxyribonukleinsäure)-Synthese schafft. Dadurch werden antiproliferative und antiinflammatorische Effekte vermittelt [48]. Für die Anwendung bei Patienten mit atopischer Dermatitis im Dosisbereich zwischen 10 und 15 (25) $\mathrm{mg} /$ Woche p.o./s.c. gibt es mittlerweile eine gute klinische Evidenz [12]. Dies trifft insbesondere für die Anwendung im Kindesalter zu [13, 49-51]. Zudem liegen direkte Studienvergleichsdaten zu CSA und AZA vor [52-54]. Unter der Anwendung von MTX werden verschiedene Arzneimittelinteraktionen und UAWs beobachtet [55, 56]. Von besonderer klinischer Bedeutung sind dabei gastrointestinale Symptome mit Übelkeit und Schwindel, die häufig auch zum Abbruch der Therapie Anlass geben. Die subkutane Applikation sollte aus pharmakokinetischen und Sicherheitsgründen der oralen Gabe vorgezogen werden [57]. Durch die Gabe von $5 \mathrm{mg}$ Folsäure 24h nach MTX-Applikation kann Blutbildungsstörungen (insbesondere Agranulozytose) vorgebeugt werden $[58,59]$. Besondere Beachtung sollte bei jungen männlichen Patienten die Beratung hinsichtlich der Reduktion der Fertilität erfahren $[60,61]$.

\section{Azathioprin}

Azathioprinwird durch das Enzym Glutathion-S-Transferase metabolisiert. Der dabei entstehende Metabolit 6-Mercaptopurin passiert die Zellmembran und wird wesentlich durch die Thio-

Hautarzt 2021 · 72:321-327 https://doi.org/10.1007/s00105-020-04720-1

(c) Der/die Autor(en) 2020

J. Wohlrab · U. Mrowietz · S. Weidinger · T. Werfel · A. Wollenberg

\section{Handlungsempfehlung zur Therapieumstellung von Immunsuppressiva auf Dupilumab bei Patienten mit atopischer Dermatitis}

\section{Zusammenfassung}

Basierend auf neuen Erkenntnissen zur molekularen Pathogenese der atopischen Dermatitis, wurde neben Glukokortikoiden und Ciclosporin mit Dupilumab nun auch eine zielgerichtete antientzündliche Systemtherapie zugelassen. Wegen ihrer Pharmakologie sind weder Glukokortikoide und Ciclosporin noch die außerhalb der Zulassung angewendeten Substanzen Methotrexat, Azathioprin und Mycophenolsäurederivate für eine Langzeittherapie geeignet. Bei der Umstellung der Therapie von den genannten niedermolekularen Substanzen auf Dupilumab sollten verschiedene Faktoren berücksichtigt werden. Hierbei sind sowohl der konkrete Anlass der Umstellung (Unwirksamkeit, unerwünschte Wirkungen oder sich einstellende Kontraindikationen) als auch die pharmakologischen Gegebenheiten zu berücksichtigen. Da es hierzu bisher keine konkreten klinischen Untersuchungen gibt, haben die Autoren auf der Grundlage einer Literaturrecherche Handlungsempfehlungen für den praktischen Alltag erarbeitet.

Schlüsselwörter

Antientzündliche Systemtherapie · Langzeittherapie - Pharmakologie . Handlungsempfehlungen · Molekulare Pathogenese

\section{Recommendations when switching therapy from immunosuppressive drugs to dupilumab in patients with atopic dermatitis}

\section{Abstract}

Based on new insights into the molecular pathogenesis of atopic dermatitis, a targeted anti-inflammatory therapy-dupilumab-has recently been approved as treatment alongside glucocorticoids and ciclosporin. Due to their pharmacology, neither glucocorticoids nor ciclosporin nor the off label used substances methotrexate, azathioprine and mycophenolic acid derivatives are suitable for longterm therapy. When switching therapy from small molecular substances to dupilumab, various factors should be considered. Both the specific cause of the change (ineffectiveness, adverse effects or contraindications) as well as the pharmacological conditions should be taken into account. Since there have been no specific clinical studies on this subject so far, the authors relied mainly on a literature search to draw up recommendations for practical everyday use.

\section{Keywords}

Anti-inflammatory systemic treatment · Longterm treatment $\cdot$ Pharmacology $\cdot$ Treatment recommendations . Molecular pathogenesis purinmethyltransferase (TPMT) weiter in verschiedene Metabolite gespalten, die die DNA- und RNA-Synthese und somit die Klonalisierung von $\mathrm{T}$ - und B-Zellen hemmen [62]. Die daraus resultierende immunsuppressive Wirkung wird in Abhängigkeit von der individuell variablen Expression des Genotyps der TPMT und deren daraus resultierender Aktivität im Dosisbereich von 1-3 mg/ kgKG/Tag AZA (in 1 bis 3 Einzeldosen) erzielt $[17,63]$. Als relevante UAWs gelten Leukozytopenie, Thrombopenie und Anämie (Myelotoxizität), Infektanfäl- ligkeit, Überempfindlichkeitsreaktionen bzw. Leber- und Nierenfunktionsstörungen sowie in Assoziation mit UV-Licht ein erhöhtes Risiko für nichtmelanozytären Hautkrebs. Männliche Patienten sollten während und bis 6 Monate nach Therapie keine Kinder zeugen [62].

\section{Mycophenolsäurederivate}

Mycophenolat-Mofetil (MMF) und Mycophenolnatrium (MPN) sind 2 Prodrugs der MPA, die ihre Wirkung durch Hemmung der Inosinmonophosphatdehydrogenase, einem bedeutsamen 
Enzym bei der DNA-Synthese, insbesondere in Lymphozyten entfaltet [64, 65]. MMF wird bei der AD im Dosisbereich von 2-mal/Tag 0,5-1,0 g p.o. und MPN 2-mal/Tag $720 \mathrm{mg}$ p.o. eingesetzt [66]. Beide Prodrugs gelten hinsichtlich ihrer Wirksamkeit in den genannten Dosisbereichen bei der AD als weitgehend äquivalent. Bezüglich potenzieller UAWs sollte v. a. die teratogene Wirkung der MPA beachtet werden [67]. Darüber hinaus können unter der Anwendung Infektionen, Blutbildungsstörungen (insbesondere Anämie und Thrombopenie) sowie gastrointestinale Symptome auftreten.

\section{Alitretinoin}

Der Einsatz von ALI wird insbesondere im Rahmen eines chronischen Handekzems praktiziert [68, 69]. Allerdings liegen auch Daten zum therapeutischen Nutzen bei AD vor [19]. Die Wirksamkeit von $\mathrm{ALI}$ bei $\mathrm{AD}$ lässt sich durch die duale agonistische Wirkung auf den Retinsäurerezeptor (RAR) und den Retinoid-X-Rezeptor (RXR) ableiten [70]. ALI wirkt wie alle Retinoide teratogen und darf nicht bei Schwangeren eingesetzt werden [71].

\section{Szenarien für einen Wechsel der Systemtherapie}

Für einen Therapiewechsel können mehrere Ursachenkonstellationen vorliegen, die ein objektives oder subjektives Hindernis zur Fortsetzung einer etablierten Therapie darstellen.

Eine ungenügende Wirksamkeit liegt dann vor, wenn nach ausreichend langer Anwendungsdauer (ca. 12 bis 16 Wochen) in einer ausreichend hohen Dosis (empfohlener Dosisbereich entsprechend Zulassung) keine oder eine zu geringe Wirksamkeit (z.B. Nichterreichen eines EASI-50) festgestellt werden muss. Zudem kann auch nach längerfristiger, wirksamer Therapie eine sekundäre Wirkminderung eintreten, die zum Therapiewechsel veranlasst. Darüber hinaus können unter der Pharmakotherapie spezifische oder unspezifische unerwünschte Wirkungen (z.B. Infektion) eintreten bzw. sich Kontraindikation für die Fortsetzung der Therapie (z.B.
Kinderwunsch, Schwangerschaft) ergeben [71]. UAWs können schwerwiegend sein und obligat einen Therapieabbruch notwendig machen. Meistens stellen sich aber befristet tolerierbare bzw. kontrollierbare UAWs ein, die dennoch einer mittel- und langfristigen Therapiefortsetzung entgegenstehen und einen Therapiewechsel begründen.

Bei der Auswahl einer klinisch und pharmakologisch fundierten Handlungsstrategie müssen mehrere Faktoren beachtet werden. Kern der Überlegungen stellt hierbei das Bemühen dar, Schaden vom Patienten abzuwenden. Die Risiken liegen dabei sowohl im Eintreten unerwünschter Arzneimittelwirkungen als auch in der Zunahme der Krankheitsaktivität (schnelles Rezidiv) während des Therapiewechsels. Im Folgenden werden typische klinische Situationen dargestellt und Handlungsempfehlungen formuliert. Die Empfehlungen basieren mangels klinischer Daten auf einer nichtsystematischen Literaturrecherche, auf pharmakologischen Erwägungen sowie auf den Erfahrungen der Experten.

\section{Grundsätzliches}

Hinsichtlich eines Therapiewechsels von kleinmolekularen Arzneistoffen (CSA, MTX, AZA oder MMF/MPN) auf einen therapeutischen Antikörper (mAb) muss zunächst grundsätzlich festgestellt werden, dass dessen Pharmakokinetik ein völlig unterschiedliches Eliminationsverhalten zugrunde liegt [42]. Während kleinmolekulare Arzneistoffe durch renale oder hepatische Elimination bzw. durch Metabolisierung in anderen Geweben inaktiviert werden, erfolgt die Degradierung von mAb v. a. durch Bindung an das Zielepitop („antigen sink“), durch Proteolyse nach Aufnahme durch Zellen des retikuloendothelialen Systems (z.B. Makrophagen) bzw. durch unspezifische Endozytose (z.B. durch Endothelzellen) und anschließende lysosomale Zersetzung. Eine chemische Interaktion zwischen kleinmolekularen Arzneistoffen und $\mathrm{mAb}$ ist somit extrem unwahrscheinlich und besitzt keine praktische Relevanz. Eine Koapplikation der beiden Substanzgruppen ist somit aus pharmakokinetischer Sicht unkritisch [72].

\section{Therapie mit Ciclosporin}

Ciclosporin (CSA) ist eine seit Langem bekannte und pharmakologisch gut charakterisierte Substanz. Multiple Studien aus der Transplantationsmedizin sowie den Phase-III- und -IV-Studien in der Indikation $\mathrm{AD}$ zeigen, dass CSA bei $\mathrm{AD}$ eine rasch wirksame und bei internistisch gesunden Patienten auch gut verträgliche Substanz darstellt. Dennoch ist bekannt, dass insbesondere bei multimorbiden bzw. älteren Patienten nach ca. 1 bis 2 Jahren Anwendungsdauer (insbesondere bei Einsatz hoher Dosen von $5,0 \mathrm{mg} / \mathrm{kgKG} / \mathrm{Tag}$ ) das Risiko der Entwicklung von UAWs relevant zunimmt. Diese Beobachtung hat zum einen dazu geführt, dass nach dem Ansprechen und Stabilisieren der Krankheitsaktivität eine Dosisreduktion auf 2,5 mg/kgKG/Tag angestrebt werden sollte, zum anderen die Empfehlung ausgesprochen wird, nach 2-jähriger Anwendung auch bei Ausbleiben von UAWs die Therapie nach Möglichkeit auf eine andere Erhaltungstherapie umzustellen [26]. Eine besondere Situation ergibt sich bei Kindern, die deutlich seltener als Erwachsene relevante UAWs entwickeln und bei denen bis zum Alter von 12 Jahren aktuell auch keine zugelassenen Therapiealternativen existieren [38].

Ciclosporin muss insbesondere bei laborchemischen Hinweisen auf eine nephrotoxische UAW in der Dosis reduziert oder abgesetzt werden. Steigt der Kreatiningehalt im Serum um mehr als $30 \%$ vom Ausgangswert (auch wenn dieser im Normbereich liegt) und persistiert über 2 Wochen, sollte die Dosis um $1 \mathrm{mg} /$ $\mathrm{kgKG} / \mathrm{Tag}$ reduziert werden. Kommt es darunter auch nach 4 Wochen zu keinem relevanten Kreatininabfall, sollte die Dosis weiter um $1 \mathrm{mg} / \mathrm{kgKG} / \mathrm{Tag}$ reduziert bzw. bei weiterer Persistenz des Kreatininanstieges abgesetzt werden. Tritt hingegen eine Kreatininsteigerung von $>50 \%$ des Ausgangswertes auf, sollte die Dosis halbiert und nach 2 bis 4 Wochen ohne Kreatininabfall abgesetzt werden. Bei bekannter Vorschädigung der Nierenfunktion sollte 


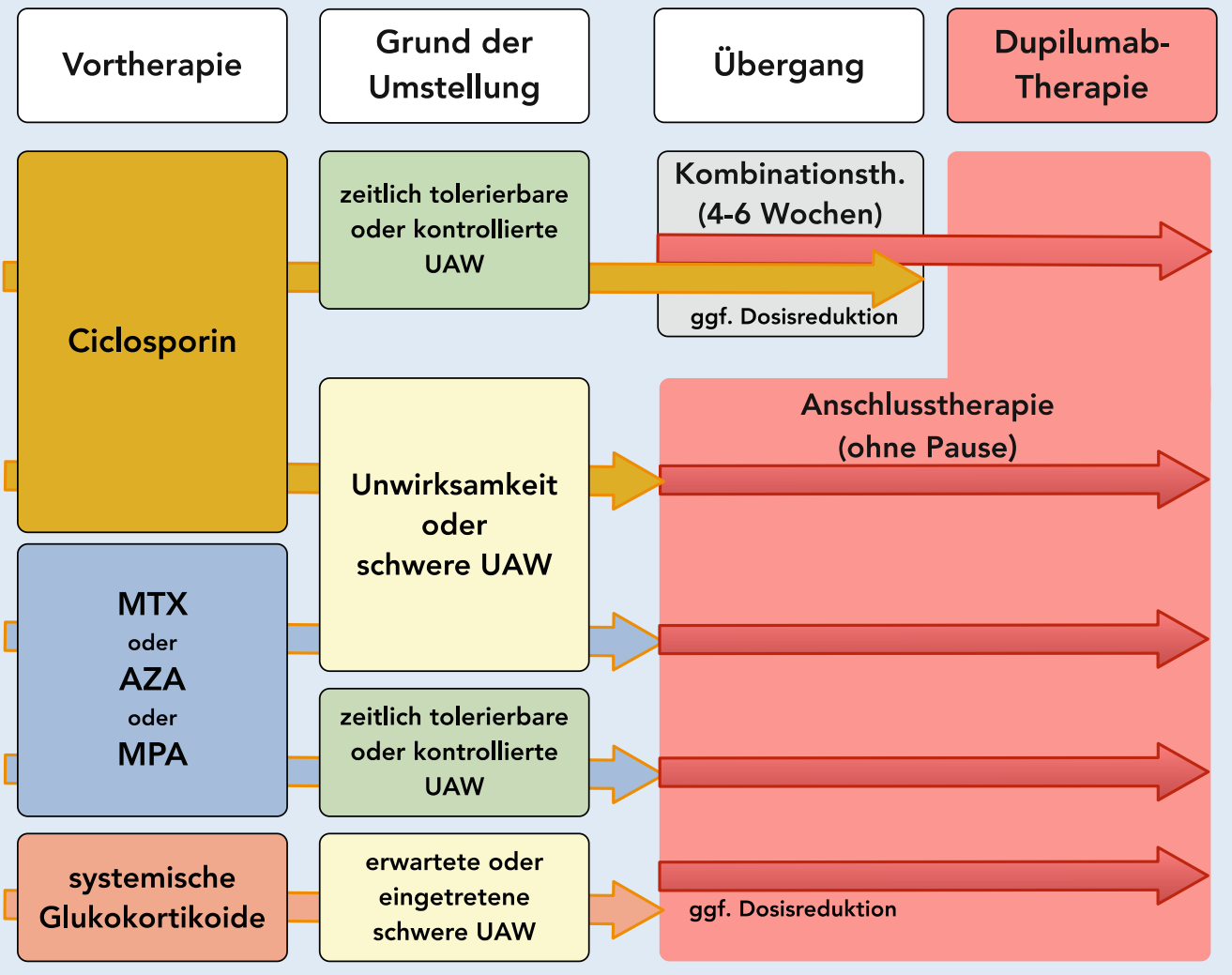

Abb. $1<$ Schematische Darstellung der Strategien zum Therapiewechsel systemischer antiinflammatorischer Therapien bei atopischer Dermatitis. MTXMethotrexat, AZA Azathioprin, MPA Mycophenolsäurederivate, UAW unerwünschte Arzneimittelwirkung

eine maximale Initialdosis von $2,5 \mathrm{mg} /$ $\mathrm{kgKG} / \mathrm{Tag}$ zur Anwendung kommen. Auch die Entwicklung einer arteriellen Hypertonie stellt eine bekannte UAW dar. Diese kann ggf. durch eine antihypertensive Therapie korrigiert werden. Stellen sich hier therapierefraktäre hypertensive Werte ein, muss die Therapie abgebrochen werden.

Für die Umstellung der Therapie auf einen $\mathrm{mAb}$ (z. B. Dupilumab) ist zu beachten, dass bei Unwirksamkeit von CSA (Nichterreichen eines EASI-50) in einer Dosierung von $5 \mathrm{mg} / \mathrm{kgKG} /$ Tag über 12 bis 16 Wochen bzw. bei Eintreten von nicht zeitlich tolerierbaren oder kompensierten UAWs CSA abgesetzt werden und sofort anschließend, ohne Therapiepause, die Einstellung auf Dupilumab erfolgen sollte (•Abb. 1). Erfolgt die Therapieumstellung auf Dupilumab aber wegen zeitlich tolerierbarer oder gar kompensierter UAWs bzw. bei einer CSAAnwendung über 1 bis 2 Jahre zur Vermeidung von UAWs, sollte CSA in der etablierten Dosis für 4 bis 6 Wochen überlappend zur Dupilumab-Therapie gege- ben werden, um ein schnelles Rezidiv der $\mathrm{AD} z u$ vermeiden (• Abb. 1$)$.

\section{Therapie mit Methotrexat, Azathioprin oder Mycophenol- säurederivaten}

Bei den nicht zur Behandlung der AD zugelassenen Arzneistoffen MTX, AZA und MMF/MPN sollte beachtet werden, dass bei Unwirksamkeit bzw. beim Auftreten relevanter UAWs ein Absetzen ohne Ausschleichen erfolgt. Auch hier ist unmittelbar im Anschluss (ohne Therapiepause) der Beginn einer Dupilumab-Applikation möglich. Eine zeitliche Überlappung durch Kombinationsgabe über 4 bis 6 Wochen ist zwar pharmakokinetisch unkritisch, erscheint aber wegen der längeren Effekthalbwertszeiten nicht sinnvoll, da ein schnelles Rezidiv nicht zu erwarten ist. Anders ist der Umstand zu bewerten, wenn im Vorfeld noch keine CSA-Gabe erfolgte und ein Umstellen auf CSA angestrebt wird.

\section{Korrespondenzadresse}

\section{Prof. Dr. Johannes Wohlrab}

Universitätsklinik und Poliklinik für

Dermatologie und Venerologie, Martin-LutherUniversität Halle-Wittenberg

Ernst-Grube-Str. 40, 06097 Halle (Saale), Deutschland

johannes.wohlrab@medizin.uni-halle.de

Funding. Open Access funding enabled and organized by Projekt DEAL.

\section{Einhaltung ethischer Richtlinien}

Interessenkonflikt. Alle Autoren haben von Sanofi Honorare für Vorträge und/oder Beratung und/ oder Mittel für wissenschaftliche Projekte und/oder klinische Studien erhalten.

Für diesen Beitrag wurden von den Autoren keine Studien an Menschen oder Tieren durchgeführt. Für die aufgeführten Studien gelten die jeweils dort angegebenen ethischen Richtlinien.

Open Access. Dieser Artikel wird unter der Creative Commons Namensnennung 4.0 International Lizenz veröffentlicht, welche die Nutzung, Vervielfältigung, Bearbeitung, Verbreitung und Wiedergabe in jeglichem Medium und Format erlaubt, sofern Sie den/die ursprünglichen Autor(en) und die Quelle ordnungsgemäß nennen, einen Link zur Creative Commons Lizenz 
beifügen und angeben, ob Änderungen vorgenommen wurden.

Die in diesem Artikel enthaltenen Bilder und sonstiges Drittmaterial unterliegen ebenfalls der genannten Creative Commons Lizenz, sofern sich aus der Abbildungslegende nichts anderes ergibt. Sofern das betreffende Material nicht unter der genannten Creative Commons Lizenz steht und die betreffende Handlung nicht nach gesetzlichen Vorschriften erlaubt ist, ist für die oben aufgeführten Weiterverwendungen des Materials die Einwilligung des jeweiligen Rechteinhabers einzuholen.

Weitere Details zur Lizenz entnehmen Sie bitte der Lizenzinformation auf http://creativecommons.org/ licenses/by/4.0/deed.de.

\section{Literatur}

1. Fishbein AB, Mueller K, Lor J, Smith P, Paller AS, Kaat A (2019) Systematic review and metaanalysis comparing topical corticosteroids with vehicle/moisturizer in childhood atopic dermatitis. JPediatr Nurs 47:36-43

2. Falk ES (1985) UV-light therapies in atopic dermatitis. Photodermatol 2:241-246

3. Legat FJ, Hofer A, BrabekE, Quehenberger F, Kerl H, Wolf $P$ (2003) Narrowband UV-B vs medium-dose UV-A1 phototherapy in chronic atopic dermatitis. Arch Dermatol 139:223-224

4. Dayal S, Pathak K, Sahu P, Jain VK (2017) Narrowband UV-B phototherapy in childhood atopic dermatitis: efficacy and safety. An Bras Dermatol 92:801-806

5. Wollenberg A, Oranje A, Deleuran Met al (2016) European Task Force on Atopic Dermatitis/EADV Eczema Task Force. ETFAD/EADV Eczema task force 2015 position paper on diagnosis and treatment of atopic dermatitis in adult and paediatric patients. JEur Acad Dermatol Venereol 30:729-747

6. Hajar T, Leshem YA, Hanifin JM, Nedorost ST, Lio PA, Paller AS, Block J, Simpson EL (2015) A systematic review of topical corticosteroid withdrawal ("steroid addiction") in patients with atopic dermatitis and other dermatoses. J Am Acad Dermatol 72:541-549e2

7. Schafer-Korting M, Schmid MH, Korting HC (1996) Topical glucocorticoids with improved risk-benefit ratio. Rationale of a new concept. Drug Saf 14:375-385

8. Luger T, Loske KD, Elsner P et al (2004) Topical skin therapy with glucocorticoids - therapeutic index. JDtsch Dermatol Ges 2:629-634

9. Hong $\mathrm{CH}$, Gooderham M, Bissonnette R (2019) Evidence review of topical Calcineurin inhibitors for the treatment of adult atopic dermatitis. JCutan Med Surg 23:5S-10S

10. Schmitt J, Schmitt N, Meurer M (2007) Cyclosporin in the treatment of patients with atopic eczema-a systematic review and meta-analysis. J Eur Acad Dermatol Venereol 21:606-619

11. Thibodeaux Q, Smith MP, Ly K, Beck K, Liao W, Bhutani T (2019) A review of dupilumab in the treatment of atopic diseases. Hum Vaccin Immunother 15:2129-2139

12. Deo M, Yung A, Hill S, Rademaker M (2014) Methotrexate for treatment of atopic dermatitis in children and adolescents. Int J Dermatol 53:1037-1041

13. TaiebY,BaumS, AmitaiBD, Barzilai A, Greenberger S (2019) The use of methotrexate for treating child- hood atopic dermatitis: a multicenter retrospective study. J Dermatolog Treat 30:240-244

14. Meggitt SJ, Reynolds NJ (2001) Azathioprine for atopic dermatitis. Clin Exp Dermatol 26:369-375

15. Hughes R, Collins P, Rogers S (2008) Further experience of using azathioprine in the treatment of severe atopic dermatitis. Clin Exp Dermatol 33:710-711

16. Schram ME, Roekevisch E, Leeflang MM, Bos JD, Schmitt J, Spuls PI (2011) A randomized trial of methotrexate versus azathioprine for severe atopic eczema. J Allergy Clin Immunol 128:353-359

17. Caufield M, Tom WL (2013) Oral azathioprine for recalcitrant pediatric atopic dermatitis: clinical response and thiopurine monitoring. J Am Acad Dermatol 68:29-35

18. Thijs JL, Van Der Geest BAM, Van Der Schaft J et al (2017) Predicting therapy response to mycophenolic acid using UGT1 A9 genotyping: towards personalized medicine in atopic dermatitis. J Dermatolog Treat 28:242-245

19. Grahovac M, Molin S, Prinz JC, Ruzicka T, Wollenberg A (2010) Treatment of atopic eczema with oral alitretinoin. Br J Dermatol 162:217-218

20. Ludwig CM, Krase JM, Price KN, Lio PA, Shi VY (2019) A practical guide for transitioning from classical immunosuppressants to dupilumab in atopic dermatitis. J Dermatolog Treat. https://doi org/10.1080/09546634.2019.1682498

21. Lee SS, Tan AW, Giam YC (2004) Cyclosporin in the treatment of severe atopic dermatitis: a retrospective study. Ann Acad Med Singapore 33:311-313

22. Arvas A (2014) Vaccination in patients with immunosuppression. Turk Pediatri Ars 49:181-185

23. Paziana K, Del Monaco M, Cardonick E, Moritz M, Keller M, Smith B, Coscia L, Armenti V (2013) Ciclosporin use during pregnancy. Drug Saf 36:279-294

24. Ellison JA, Patel L, Ray DW, David TJ, Clayton PE (2000) Hypothalamic-pituitary-adrenal function and glucocorticoid sensitivity in atopic dermatitis. Pediatrics 105:794-799

25. Khan YK, Kalaaji AN, Clarke BL (2008) Glucocorticoid-induced osteoporosis in dermatologic practice: a review. JDrugs Dermatol 7:1053-1059

26. European Dermatology Forum tEAoD, Venereology tEAoA, Clinical Immunology tETFoADEFoA, Airways Diseases Patients' Associations tESfD, Psychiatry tESoPDGA, Asthma European N, European Union of Medical S., Wollenberg A, BarbarotS Bieber T et al (2018) Consensus-based European guidelines for treatment of atopic eczema (atopic dermatitis) in adults and children: part Il. J Eur Acad Dermatol Venereol 32:850-878

27. European Dermatology Forum tEAoD, Venereology tEAoA, Clinical Immunology tETFoADEFoA, Airways Diseases Patients' Associations tESfD, Psychiatry tESoPDGA, Asthma European N, European Union of Medical S., Wollenberg A, BarbarotS Bieber T et al (2018) Consensus-based European guidelines for treatment of atopic eczema (atopic dermatitis) in adults and children: part I. J Eur Acad Dermatol Venereol 32:657-682

28. Faulds D, Goa KL, Cyclosporin BP (1993) A review of its pharmacodynamic and pharmacokinetic properties, and therapeutic use in immunoregulatory disorders. Drugs 45:953-1040

29. Tedesco D, Haragsim L (2012) Cyclosporine: a review. J Transplant. https://doi.org/10.1155/ 2012/230386

30. Hojo M, Morimoto T, Maluccio M, Asano T, Morimoto K, Lagman M, Shimbo T, Suthanthiran M (1999)
Cyclosporine induces cancer progression by a cellautonomous mechanism. Nature 397:530-534

31. Muellenhoff MW, Koo JY (2012) Cyclosporine and skin cancer: an international dermatologic perspective over 25 years of experience. A comprehensive review and pursuit to define safe use of cyclosporine in dermatology. J Dermatolog Treat 23:290-304

32. Marcil I, Stern RS (2001) Squamous-cell cancer of the skin in patients given PUVA and ciclosporin: nested cohort crossover study. Lancet 358:1042-1045

33. Cifkova R, Hallen H (2001) Cyclosporin-induced hypertension. JHypertens 19:2283-2285

34. Keown PA, Stiller CR, Ulan RA (1981) Use of cyclosporin a despite renal dysfunction. $\mathrm{N}$ Engl J Med 305:227-228

35. Sturrock ND, Lang CC, Struthers AD (1993) Cyclosporin-induced hypertension precedes renal dysfunction and sodium retention in man. JHypertens 11:1209-1216

36. V'Lckova-Laskoska MT (2005) Cyclosporin A-induced gingival hyperplasia in psoriasis: review of the literature and case reports. Acta Dermatovenerol Croat 13:108-113

37. Leonardi S, Marchese G, Rotolo N, Miraglia Del Giudice M, La Rosa M (2004) Cyclosporin is safe and effective in severe atopic dermatitis of childhood. Report of three cases. Minerva Pediatr 56:231-237

38. Harper Jl, Berth-Jones J, Camp RD, Dillon MJ, Finlay AY, Holden CA, O'Sullivan D, Veys PA (2001) Cyclosporin for atopic dermatitis in children. Dermatology 203:3-6

39. Yee J, Orchard D (2018) Monitoring recommendations for oral azathioprine, methotrexate and cyclosporin in a paediatric dermatology clinic and literature review. Australas J Dermatol 59:31-40

40. Andrews DJ, Cramb R (2002) Cyclosporin: revisions in monitoring guidelines and review of current analytical methods. Ann Clin Biochem 39:424-435

41. D'Ippolito D, Pisano M (2018) Dupilumab (Dupixent): an interleukin-4 receptor antagonist for atopic dermatitis. PT 43:532-535

42. Wohlrab J (2015) Pharmacokinetic characteristics of therapeutic antibodies. J Dtsch Dermatol Ges 13:530-534

43. EMA Assessment Report Dupixent (EMA/512262/ 2017). https://www.ema.europa.eu/en/ documents/assessment-report/dupixent-eparpublic-assessment-report_en.pdf Zugegriffen: 27. Jan. 2020

44. Blauvelt A, de Bruin-Weller M, Gooderham $M$ et al (2017) Long-term management of moderateto-severe atopic dermatitis with dupilumab and concomitant topical corticosteroids (LIBERTY AD CHRONOS): a 1-year, randomised, doubleblinded, placebo-controlled, phase 3 trial. Lancet 389:2287-2303

45. Thomson J, Wernham AGH, Williams HC (2018) Long-term management of moderate-to-severe atopic dermatitis with dupilumab and concomitant topical corticosteroids (LIBERTY AD CHRONOS): a critical appraisal. Br J Dermatol 178:897-902

46. Wollenberg $A$, Beck LA, Blauvelt A, Simpson EL, Chen Z, Chen Q, Shumel B, Khokhar FA, Hultsch T, Rizova E, Rossi AB, Graham NMH, Pirozzi G, Lu Y, Ardeleanu M (2020) Laboratory safety of dupilumab in moderate-to-severe atopic dermatitis: results from three phase III trials (LIBERTY AD SOLO 1, LIBERTY AD SOLO 2, LIBERTY ADCHRONOS). Br JDermatol 182:1120-1135

47. Deleuran M, Thaçi D, Beck LA et al (2020) Dupilumab shows long-term safety and efficacy in 
patients with moderate to severe atopic dermatitis enrolled in a phase 3 open-label extension study. JAm Acad Dermatol 82:377-388

48. Johnston A, Gudjonsson JE, Sigmundsdottir $H$, Ludviksson BR, Valdimarsson $\mathrm{H}$ (2005) The antiinflammatory action of methotrexate is not mediated by lymphocyte apoptosis, but by the suppression of activation and adhesion molecules. Clin Immunol 114:154-163

49. Dvorakova V, O'Regan GM, Irvine AD (2017) Methotrexate for severe childhood atopic dermatitis: clinical experience in a tertiary center. Pediatr Dermatol 34:528-534

50. Park YW, Yeom KB, Kim KH (2013) Refractory atopic dermatitis in childhood: improvement with methotrexate? Ann Dermatol 25:114-116

51. Purvis D, Lee M, Agnew K, Birchall N, Dalziel SR (2019) Long-term effect of methotrexate for childhood atopic dermatitis. J Paediatr Child Health 55:1487-1491

52. El-Khalawany MA, Hassan H, Shaaban D, Ghonaim N, Eassa B (2013) Methotrexate versus cyclosporine in the treatment of severe atopic dermatitis in children: a multicenter experience from Egypt. Eur JPediatr 172:351-356

53. Gerbens LAA, Hamann SAS, Brouwer MWD, Roekevisch E, Leeflang MMG, Spuls PI (2018) Methotrexate and azathioprine for severe atopic dermatitis: a 5-year follow-up study of a randomized controlled trial. Br JDermatol 178:1288-1296

54. Goujon C, Viguier M, Staumont-Salle D, Bernier C, Guillet G, Lahfa M, Ferrier Le Bouedec MC, Cambazard F, Bottigioli D, Grande S, Dahel K, Berard F, Rabilloud M, Mercier C, Nicolas JF (2018) Methotrexate versus Cyclosporine in adults with moderate-to-severe atopic dermatitis: a phase III randomized noninferiority trial. J Allergy Clin Immunol Pract 6:562-69e3

55. Khan ZA, Tripathi R, Mishra B (2012) Methotrexate: a detailed review on drug delivery and clinical aspects. Expert Opin Drug Deliv 9:151-169

56. Bourre-Tessier J, Haraoui B (2010) Methotrexate drug interactions in the treatment of rheumatoid arthritis: a systematic review. J Rheumatol 37:1416-1421

57. Alsaeedi S, Keystone EC (2014) Therapy: oral or subcutaneous methotrexate for rheumatoid arthritis? Nat Rev Rheumatol 10:578-579

58. Cline A, Jorizzo JL (2017) Does daily folic acid supplementation reduce methotrexate efficacy? Dermatol Online J 23(11):13030/qt4hf5v2vk. https://pubmed.ncbi.nlm.nih.gov/29447631/

59. Prey S, Paul C (2009) Effect of folic or folinic acid supplementation on methotrexate-associated safety and efficacy in inflammatory disease: a systematic review. Br J Dermatol 160:622-628

60. Grosen A, Kelsen J, Hvas CL, Bellaguarda E, Hanauer SB (2017) The influence of methotrexate treatment on male fertility and pregnancy outcome after paternal exposure. Inflamm Bowel Dis 23:561-569

61. Gutierrez JC, Hwang K (2017) The toxicity of methotrexate in male fertility and paternal teratogenicity. Expert Opin Drug Metab Toxicol 13:51-58

62. Arnott ID, Watts D, Satsangi J (2003) Azathioprine and anti-TNF alpha therapies in Crohn's disease: a review of pharmacology, clinical efficacy and safety. Pharmacol Res 47:1-10

63. Noguera-Morel L, Knopfel N, Torrelo A, HernandezMartin A (2019) A retrospective study of systemic treatment of severe atopic dermatitis with azathioprine: effectiveness and tolerance in 11 pediatric patients. Actas Dermosifiliogr 110:227-231
64. Staatz CE, Tett SE (2014) Pharmacology and toxicology of mycophenolate in organ transplant recipients: an update. Arch Toxicol 88:1351-1389

65. Brunet M, Crespo M, Millan O, Seron D, Torregrosa V, Jimenez O, Moreso F, Martorell J, Grinyo JM, Oppenheimer F (2007) Pharmacokinetics and pharmacodynamics in renal transplant recipients under treatment with cyclosporine and Myfortic. Transplant Proc 39:2160-2162

66. Phan K, Smith SD (2020) Mycophenolate mofetil and atopic dermatitis: systematic review and metaanalysis. JDermatolog Treat 31:810-814

67. Anderka MT, Lin AE, Abuelo DN, Mitchell AA, Rasmussen SA (2009) Reviewing the evidence for mycophenolate mofetil as a new teratogen: case report and review of the literature. Am J Med Genet A 149A:1241-1248

68. D'Erme AM, Milanesi N, Agnoletti AF, Maio V, Massi D, Gola M (2014) Efficacy of treatment with oral alitretinoin in patient suffering from lichen simplex chronicus and severe atopic dermatitis of hands. Dermatol Ther 27:21-23

69. Blair HA, Alitretinoin SLJ (2016) A review in severe chronic hand eczema. Drugs 76:1271-1279

70. Cheng C, Michaels J, Scheinfeld N (2008) Alitretinoin: a comprehensive review. Expert Opin Investig Drugs 17:437-443

71. Vestergaard C, Wollenberg A, Barbarot $S$ et al (2019) European task force on atopic dermatitis position paper: treatment of parental atopic dermatitis during preconception, pregnancy and lactation period. J Eur Acad Dermatol Venereol 33:1644-1659

72. Ohtsuki M, Morita A, Igarashi A, Imafuku S, Tada Y, Fujita H, Fujishige A, Yamaguchi M, Teshima R, Tani Y, Nakagawa H (2017) Secukinumab improves psoriasis symptoms in patients with inadequate response to cyclosporine $A$ : a prospective study to evaluate direct switch. J Dermatol 44:1105-1111

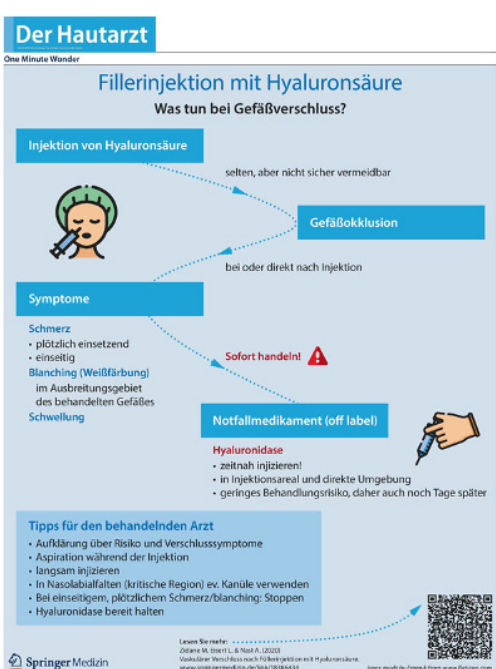

Zum One Minute Wonder dieser Ausgabe

In diesem Heft haben wir ein One Minute

Wonder aus dem Bereich der Ästhetischen

Dermatologie für Sie:

Fillerinjektion mit Hyaluronsäure:

Was tun bei Gefäßverschluss?

(www.springermedizin.de/link/18943792)

One Minute Wonder zum Beitrag:

Zidane M, Eisert L, Nast A (2021) Vaskulärer Verschluss nach Füllerinjektion mit Hyaluronsäure. Hautarzt 72: 329-331 www.springermedizin.de/link/18386434

Erstellt im Februar 2021 von:

Dr. J. Kind, Heidelberg

Beratung: PD Dr. Dr. A. Zink, München

Geprüft und freigegeben im März 2021

von: Dr. M. Zidane, Berlin, und

PD Dr. Dr. A. Zink, München

Gestaltung: U. Stöcklein, le-tex Leipzig

Haben Sie Fragen oder Anregungen zu diesem One Minute Wonder?

Die Redaktion freut sich auf regen Austausch (julie.kind@springer.com). 